\title{
Candidate single nucleotide polymorphisms of irritable bowel syndrome: a systemic review and meta- analysis
}

Shiwei Zhu, Ben Wang, Qiong Jia and Liping Duan*

\begin{abstract}
Background: Genetic factors increase the risk of irritable bowel syndrome (IBS). Analysis of single nucleotide polymorphisms (SNPS) has been used in IBS patients, but the findings are inconsistent. The goal of this review was to synthesize all the published SNPs studies of IBS through meta-analysis to objectively evaluate the relevance of SNPs to IBS risks.

Methods: IBS - related polymorphisms studies from 2000 to 2018 were searched. Pooled odds ratios with a 95\% confidence interval for each SNP were evaluated through five genetic models. Ethnicity, ROME criteria and IBS subtypes were defined for subgroup analyze.

Results: Ten relevant genes were evaluated. SNPs rs4263839 and rs6478108 of TNFSF15 associated with an increased risk of IBS; IL6 rs 1800795 increased the risk for Caucasian IBS patients which diagnosed by Rome III criteria; and IL23R rs11465804 increased the risk for IBS-C patients. IL10 rs1800896 GG genotype associated with a decreased risk of IBS. No evidence supported the association of GNB3 rs5443, TNFa rs1800629, and IL10 rs1800871 to IBS in this study.

Conclusions: This meta-analysis presents an in-depth overview for IBS SNPS analysis. It was confirmed that polymorphisms of TNFSF15 associated with increased IBS risk, while IL10 rs1800896 associated with decreased IBS risk. It might offer some insights into polymorphisms of inflammation factors which might affect IBS susceptibility. Moreover, the analysis also emphasizes the importance of diagnostic criteria and phenotype homogeneity in IBS genetic studies.
\end{abstract}

Keywords: Genetic risk, Irritable bowel syndrome, Single nucleotide polymorphisms, TNFSF15 IL10

\section{Background}

Irritable bowel syndrome (IBS) is a predominant and common chronic gastrointestinal (GI) disorder presenting with recurrent abdominal pain accompanied with altered bowel habits. IBS has been a continually increasing trend worldwide, especially in developing countries. It leads to negative effects on the quality of life and the work efficiency of affected patients. According to the Rome IV criteria, IBS is categorized into four subtypes [1], diarrhea predominant IBS (IBS-D), constipation predominant IBS (IBS-C), mixture of diarrhea and constipation IBS (IBS-M) and un-subtyped IBS (IBS-U).

* Correspondence: duanlp@bjmu.edu.cn

Department of Gastroenterology, Peking University Third Hospital, No.49 North Garden Rd., Haidian District, Beijing 100191, China
Genetic, environmental and psychological factors, which may result in "brain-gut-axis" dysfunction [2], increase the risk of IBS. In addition, the consequential pathophysiological mechanisms [3] such as changes in gastrointestinal motility, visceral hypersensitivity, increasing mucosal permeability, immune activation and gut microbiota dysbiosis, are evaluated in many researches. Due to the multifactorial origin and the elusive etiology of IBS, there is no consensus on diagnostic biomarkers/methods or curative therapy it.

In early 2000, twins [4] and family [5, 6] studies demonstrated a more heritable component to IBS. The associations of IBS and its risk gene polymorphisms have been ascertained by many researchers. Single nucleotide polymorphisms (SNPs) represent the most widespread type of sequence variations in genomes. It is known to

(c) The Author(s). 2019 Open Access This article is distributed under the terms of the Creative Commons Attribution 4.0 International License (http://creativecommons.org/licenses/by/4.0/), which permits unrestricted use, distribution, and reproduction in any medium, provided you give appropriate credit to the original author(s) and the source, provide a link to the Creative Commons license, and indicate if changes were made. The Creative Commons Public Domain Dedication waiver (http://creativecommons.org/publicdomain/zero/1.0/) applies to the data made available in this article, unless otherwise stated. 
be valuable genetic markers, because it may reveal the evolutionary history and common genetic polymorphisms that explain the hereditary risks for common diseases such as inflammation bowel disease (IBD) [7, 8]. Case-control studies have examined the possible role of different SNPs in patients with IBS, such as serotonin transporter protein (SERT) [9], Catechol-Omethyltransferase (COMT) [10], $\beta 3$ subunit of Gprotein (GN $\beta 3)$ [11], voltage-gated mechanosensitive $\mathrm{Na}(+)$ channel NaV1.5 (SCN5A) [12], and tumor necrosis factor (TNF)- $\alpha$ [13]. Some meta-analysis previously were conducted and researchers attempted to extract commonalities as well. Owing to unclear or mixed ethnicity, patients' population changes, updating of Rome diagnostic criteria and usage of different genetic models, conclusion of association for SNPs and IBS have still been inconsistent over time.

Therefore, this systematic review aimed to synthesize and updated previous SNPs studies through meta-analysis, in order to produce an in-depth analysis of genetic SNPs with IBS from a more detailed perspective.

\section{Methods}

\section{Search strategy and study selection}

Studies of irritable bowel syndrome and its associated genetic polymorphisms were identified by systematically searching from the following databases: PubMed, Web of Science, EMBASE, Cochrane Clinical Trials Database, Medline and Chinese database Chinese National Knowledge Infrastructure. Searching terms of medical subject headings $(\mathrm{MeSH})$ included 'irritable bowel syndrome, IBS' combined with 'polymorphism, genetic polymorphism, single nucleotide polymorphisms, SNPs'. Studies were concerned in the period of 2000.01-2018.01 and the search was not limited by language or publication status. Potentially relevant articles were screened by at least 2 independent reviewers, and disagreements were resolved by discussion or input from a third reviewer if required.

\section{Inclusion criteria and quality assessment}

All candidate studies were included if they met all the inclusion criteria as follow: (i) Case-control studies with subjects' information, available allele frequency and no consanguinity between the case and control groups. (ii) Explicit ethnicity such as Caucasian or Mongolian. (iii) IBS diagnosis based on clinical examination and specific diagnostic criteria such as Rome I-III. (iv) Allele frequency meets Hardy-Weinberg equilibrium in healthy controls. (v) Largest sample size was included in reused data. Newcastle-Ottawa Quality Assessment Scale (NOS) scored as quality assessment in all studies. To confirm the test effect, SNPs that had been reported in less than 3 studies were excluded in this meta-analysis.

\section{Data extraction}

Two investigators independently extracted data from the identified publications, including the first author's name, year of publication, source of publication, IBS diagnostic criteria, DNA extraction and genotyping method, numbers and source of patients and controls, genotype frequency, and allele frequency. Discrepancies in data extraction were resolved by repeating the study review and discussing the results. The corresponding author was contacted, and genotype frequencies were requested when missing from the studies.

\section{Statistical analysis}

Hardy-Weinberg equilibrium (HWE) analysis of the controls was performed using the Chi-square test. To determine the overall gene effect, five genetic models including allele (AM), dominant (DM), recessive (RM), homozygous (HoM) and heterozygous (HeM) models were used to evaluate the allele and genotype risks for IBS $[14,15]$. Relative risks of IBS were estimated according to odds ratios (ORs) with 95\% confidence intervals (CIs). The heterogeneity among studies was assessed using the Cochran Q test [16]. The inconsistency index $\mathrm{I}^{2}$ was also calculated to quantify heterogeneity. A fixedeffects model was used to pool the results if the result of the heterogeneity test was not significant $(P>0.05)$ or $\left.\mathrm{I}^{2}<50 \%\right)$; otherwise, a random-effect model was selected. Sensitive test was conducted to determine the source of heterogeneity. Publication bias was examined by using the Begg's test only if analyzed studies were more than five [17]. Subgroups of ethnicity, diagnostic criteria and IBS subtypes were conducted in each SNP. All statistical tests were two-tailed, and the level of significance was set at $P<0.05$. STATA version 13 (Stata Corporation, College Station, TX, United States) was used for all analysis.

\section{Results \\ Study selection and characteristics analysis}

From the databases, 3810 potentially relevant publications were identified. Figure 1 shows the flow diagram of this study. After screening and inclusion, 66 SNPs were identified in IBS patients (see Additional file 1: Table S1). Finally, 10 different SNPs from 28 studies were determined; references for these studies are provided in Additional file 1: Table S1. The identified SNPs focus on neurotransmitter system (SLC6A4 5-HTTLPR, COMT rs 4680 and GNB3 rs5443) and the inflammation system (TNFa rs1800629, IL10 rs1800896, IL10 rs1800871, IL6 rs1800795, IL23R rs11465804, TNFSF15 rs4263839 and TNFSF15 rs6478108). Most of the studies reported that the DNA was extracted from blood, except for 2 studies that had DNA extracted from buccal epithelial cells and 


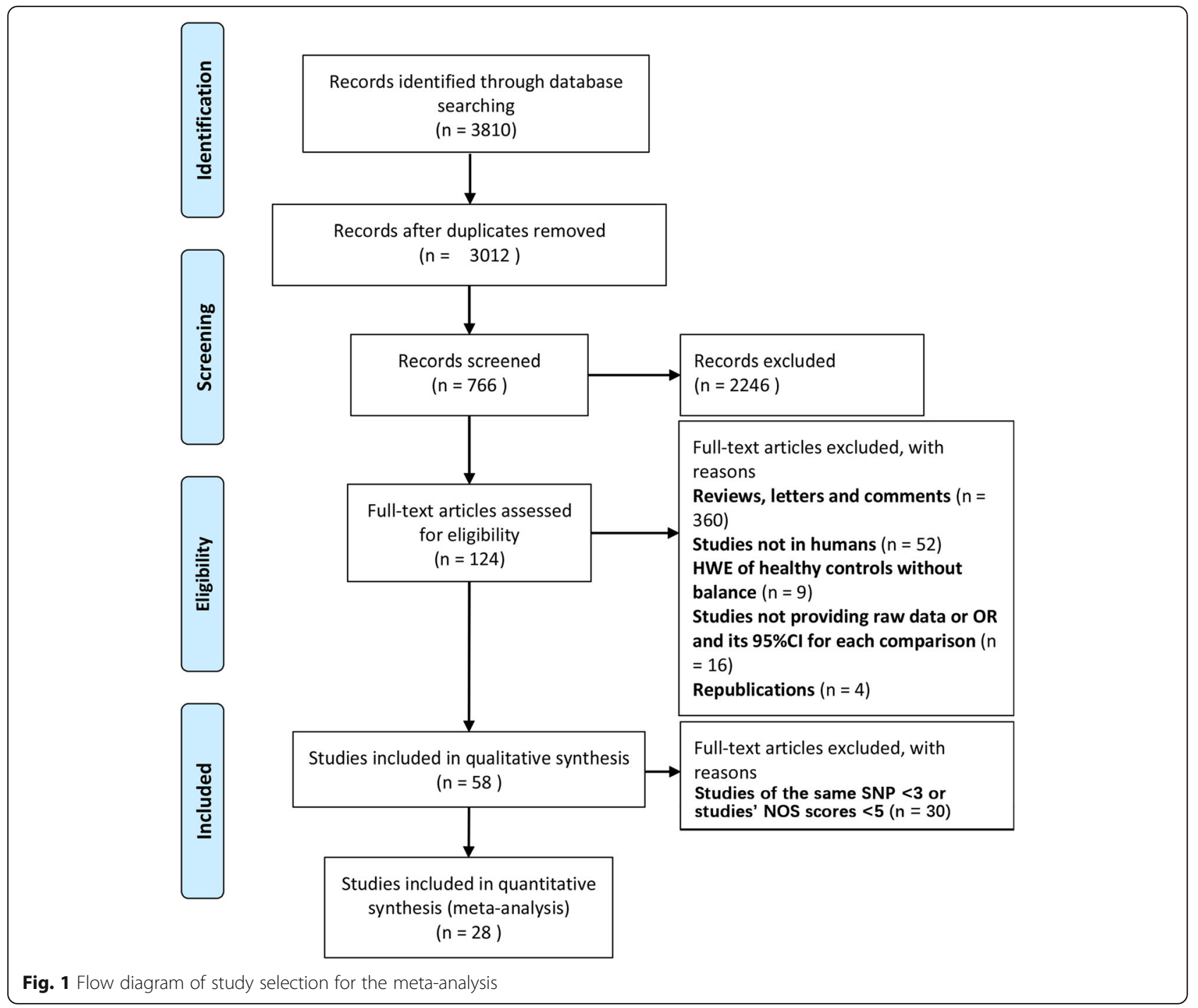

sputum. SNPs were assayed through PCR. The characteristics of these studies are shown in Additional file 1: Table S2. Rome III criteria was used in half of the studies (50\% Rome III, 46.6\% Rome II and 0.04\% Rome I). Multiple comparisons of identified SNPs through five genetic models is summarized in Table 1.

\section{SLC6A4 5-HTTLPR and IBS risk}

Twelve studies involving 1834 IBS subjects and 1941 controls were analyzed to determine the association of the SLC6A4 5-HTTLPR and IBS risk (Table 1). Genotype $l s$ presented an increased risk for IBS development in HeM ( $l s$ vs. $l l, \mathrm{OR}=2.312,95 \% \mathrm{CI}: 1.084-4.931, P=0.03$ ) (Fig. 2a). Heterogeneity for included studies is significant $(P<0.05)$. A sensitivity analysis, after excluding studies in turn, indicated that the associations remained (Fig. 2b). Begg's test suggested no publication bias $(P=0.064)$. Thus, subgroup analysis based on ethnicity or diagnostic criteria was performed. Figure $2 \mathrm{a}$ shows that polymorphism was significantly correlated with IBS risk in Mongoloid population $(\mathrm{OR}=20.68,95 \% \mathrm{CI}: 3.21-133.44, P=0.001)$, but there was no association in Caucasian populations. No significant association was found in diagnostic criteria subgroups. Further, IBS subtypes (IBS-A, IBS-C and IBS-D) were analyzed but no association was found.

\section{COMT rs4680 and IBS risk}

Three studies involving 414 IBS patients and 1363 controls were analyzed for the association of COMT rs4680 (G > A) and IBS risk (Table 1). GA genotype presented a decreased risk for IBS in the HeM (GA vs. GG, $\mathrm{OR}=0.673,95 \% \mathrm{CI}: 0.5-0.907, P=0.009$ ) (Fig. 3a). Included studies were with a good homogeneity $\left(\mathrm{I}^{2}=\right.$ 
Table 1 Summary of results of all polymorphisms for five genetic models

\begin{tabular}{|c|c|c|c|c|c|}
\hline Gene rs. & Gene Model & p1 value ${ }^{a}$ & OR $(96 \% C l)^{b}$ & p2 value & $\begin{array}{l}\text { Analysis } \\
\text { model }\end{array}$ \\
\hline \multirow[t]{5}{*}{ SLC6A4 5-HTTLPR } & AM: s vs. I & 0.008 & $1.169(0.908,1.505)$ & 0.226 & REM \\
\hline & DM: Is + ss vs. II & 0.013 & $0.967(0.689,1.358)$ & 0.848 & REM \\
\hline & RM: ss vs. II + Is & 0.008 & $1.169(0.908,1.505)$ & 0.226 & REM \\
\hline & HoM: ss vs. II & 0.004 & $1.114(0.724,1.714)$ & 0.623 & REM \\
\hline & HeM: Is vs. II & 0 & $2.312(1.084,4.931)$ & 0.03 & REM \\
\hline \multirow[t]{5}{*}{ COMT rs4680 } & AM: A vs. G & 0.07 & $1.011(0.843,1.212)$ & 0.91 & FEM \\
\hline & DM: GA + AA vs. GG & 0.311 & $0.827(0.632,1.082)$ & 0.167 & FEM \\
\hline & RM: AA vs. GG + GA & 0.044 & $1.461(0.730,2.924)$ & 0.284 & REM \\
\hline & HoM: AA vs. GG & 0.018 & $1.291(0.522,3.189)$ & 0.581 & REM \\
\hline & HeM: GA vs.GG & 0.141 & $0.673(0.5,0.907)$ & 0.009 & FEM \\
\hline \multirow[t]{5}{*}{ TNFa rs1800629 } & AM: A vs. G & 0.301 & $0.95(0.831,1.086)$ & 0.453 & FEM \\
\hline & DM: GA + AA vs. GG & 0.315 & $0.866(0.576,1.303)$ & 0.49 & FEM \\
\hline & RM: AA vs. GG + GA & 0.107 & $0.895(0.587,1.364)$ & 0.606 & FEM \\
\hline & HoM: AA vs. GG & 0.415 & $0.854(0.565,1.290)$ & 0.453 & FEM \\
\hline & HeM: GA vs.GG & 0.096 & $0.954(0.856,1.111)$ & 0.543 & FEM \\
\hline \multirow[t]{5}{*}{ IL10 rs1800896 } & AM: G vs. A & 0.774 & $0.935(0.826,1.508)$ & 0.286 & FEM \\
\hline & $\mathrm{DM}: \mathrm{GA}+\mathrm{GG}$ vs. AA & 0.842 & $1.024(0.842,1.245)$ & 0.815 & FEM \\
\hline & RM: GG vs. AA + GA & 0.321 & $0.806(0.655,0.992)$ & 0.042 & FEM \\
\hline & HoM: GG vs. AA & 0.719 & $0.855(0.661,1.105)$ & 0.23 & FEM \\
\hline & HeM: GA vs. AA & 0.659 & $1.113(0.906,1.367)$ & 0.036 & FEM \\
\hline \multirow[t]{5}{*}{ IL10 rs1800871 } & AM: C vs. T & 0.825 & $0.944(0.764,1.167)$ & 0.596 & FEM \\
\hline & DM: TC + CC vs. TT & 0.6 & $1.022(0.766,1.345)$ & 0.878 & FEM \\
\hline & RM: CC vs. $T+T C$ & 0.647 & $0.701(0.434,1.133)$ & 0.147 & FEM \\
\hline & HoM: CC vs. TT & 0.596 & $0.743(0.444,1.244)$ & 0.259 & FEM \\
\hline & HeM: TC vs.TT & 0.496 & $1.092(0.818,1.456)$ & 0.551 & FEM \\
\hline \multirow[t]{5}{*}{ IL6 rs1800795 } & AM: G vs. C & 0.039 & $1.144(0.810,1.373)$ & 0.249 & REM \\
\hline & DM: CG + GG vs. CC & 0.292 & $1.092(0.888,1.346)$ & 0.4 & FEM \\
\hline & RM: GG vs. CC + CG & 0 & $1.373(0.858,2.198)$ & 0.186 & REM \\
\hline & HoM: GG vs. CC & 0.657 & $1.099(0.872,1.387)$ & 0.657 & FEM \\
\hline & HeM: CG vs.CC & 0.038 & $0.905(0.566,1.449)$ & 0.679 & REM \\
\hline \multirow[t]{5}{*}{ IL23R rs11465804 } & AM: G vs. T & 0.005 & $1.266(0.813,1.971)$ & 0.296 & REM \\
\hline & DM: TG + GG vs. Tा & 0.004 & $1.265(0.789,2.029)$ & 0.329 & REM \\
\hline & RM: GG vs. TT+TG & 0.902 & $1.21(0.460,3.183)$ & 0.699 & FEM \\
\hline & HoM: GG vs. Tा & 0.004 & $1.244(0.770,2.009)$ & 0.372 & REM \\
\hline & HeM: TG vs.TT & 0.896 & $1.209(0.983,1.487)$ & 0.072 & FEM \\
\hline \multirow[t]{5}{*}{ TNFSF15 rs4263839 } & AM: G vs. A & 0 & $5.139(3.859,6.844)$ & 0 & REM \\
\hline & DM: GA + GG vs. AA & 0.012 & $6.527(4.616,9.229)$ & 0 & REM \\
\hline & RM: GG vs. AA+GA & 0 & $2.802(0.951,8.261)$ & 0.062 & REM \\
\hline & HoM: GG vs. AA & 0.557 & $19.127(15.395,23.765)$ & 0 & FEM \\
\hline & HeM: GA vs. AA & 0 & $9.361(4.702,18.637)$ & 0 & REM \\
\hline \multirow[t]{3}{*}{ TNFSF15 rs6478108 } & AM: T vs. C & 0.288 & $1.143(1.016,1.287)$ & 0.026 & FEM \\
\hline & DM: CT + TT vs. CC & 0.335 & $1.235(0.964,1.581)$ & 0.094 & FEM \\
\hline & RM: $\Pi T$ vs. CC + CT & 0.23 & $1.171(0.997,1.374)$ & 0.054 & FEM \\
\hline
\end{tabular}


Table 1 Summary of results of all polymorphisms for five genetic models (Continued)

\begin{tabular}{|c|c|c|c|c|c|}
\hline Gene rs. & Gene Model & p1 value ${ }^{a}$ & OR $(96 \% C l)^{b}$ & p2 value ${ }^{c}$ & $\begin{array}{l}\text { Analysis } \\
\text { model }\end{array}$ \\
\hline & HoM: TT vs. CC & 0.287 & $1.306(1.005,1.697)$ & 0.045 & FEM \\
\hline & HeM: CT vs.CC & 0.326 & $1.17(0.902,1.519)$ & 0.237 & FEM \\
\hline \multirow[t]{5}{*}{ GNß3 rs5443 } & AM: T vs. C & 0.013 & $1.167(0.825,1.651)$ & 0.383 & REM \\
\hline & DM: CT + TT vs. CC & 0.025 & $1.196(0.762,1.877)$ & 0.437 & REM \\
\hline & RM: TT vs. CC + CT & 0.227 & $1.273(0.811,1.998)$ & 0.295 & FEM \\
\hline & HoM: TT vs. CC & 0.037 & $1.394(0.701,2.772)$ & 0.344 & REM \\
\hline & HeM: CT vs.CC & 0.088 & $1.166(0.776,1.753)$ & 0.459 & FEM \\
\hline
\end{tabular}

a Cochran Q test;b Odds ratio (95\% confidence interval); c Mante-Haenszel test; AM Allele models, DM Dominant models, RM Recessive models, HoM Homozygous models, HeM Heterozygous models, REM Random effect model, FEM Fixed effect model

49\%, $P=0.141)$. Subgroups analyses were conducted but no associations were found.

\section{IL10 rs1800896 and IBS risk}

Seven studies involving 955 IBS patients and 779 controls were analyzed for the association of IL10 rs1800896 $(\mathrm{A}>\mathrm{G})$ and IBS risk (Table 1). GG genotype presented a decreased risk of IBS in the RM (GG vs. GA+AA, OR = 0.806, 95\% CI: 0.655-0.992, $P=0.042$ ) (Fig. 3b). No significant heterogeneity $\left(\mathrm{I}^{2}=14.3 \%, P=0.321\right)$ was found. Further subgroup analysis was used for ethnicity and diagnostic criteria, but no additional associations were found.

\section{IL6 rs1800795 and IBS risk}

There were four studies involving 1641 IBS patients and 1058 controls, which were analyzed for the association of IL6 rs1800795 (C> G) and IBS risk (Table 1). The data showed no association of allele or genotype with
IBS risk. The AM (G vs. C) was used for subgroup analysis. This finding was interesting because there was no association of the G allele with IBS in the Caucasian subgroup, but in Caucasian subgroups with diagnostic Rome III criteria (Fig. 3c), the IL6 rs1800795 G allele significantly increased the risk for IBS (OR $=2.057,95 \%$ CI: $1.313-3.225, P=0.002)$.

\section{IL23R rs11465804 and IBS risk}

There were four studies involving 2068 IBS patients and 1958 controls that analyzed the association of IL23R rs11465804 $(\mathrm{T}>\mathrm{G})$ and IBS risk (Table 1). The data showed no association of the polymorphism with IBS risk in any of the models. In subgroup analysis of IBS subtype, IL23R rs11465804 increased the risk for IBS-C both in AM (G vs. T, OR = 1.346, 95\% CI: $1.025-1.767$, $P=0.032)$ and DM (TG + GG vs. TT, OR $=1.338,95 \%$ CI: $1.005-1.781, P=0.046$ ) (Fig. 3d). No association was found in IBS-D patients or other subgroup.

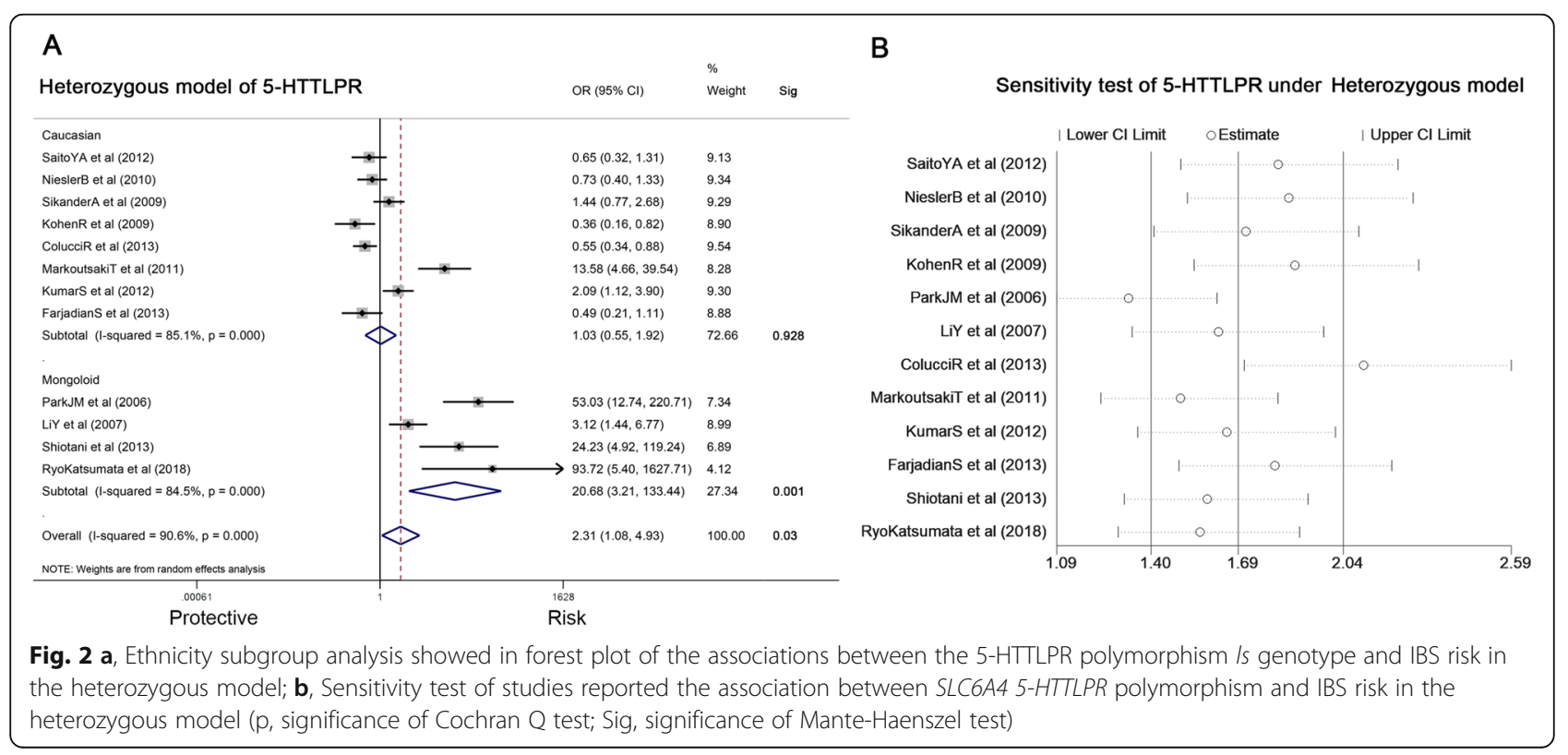




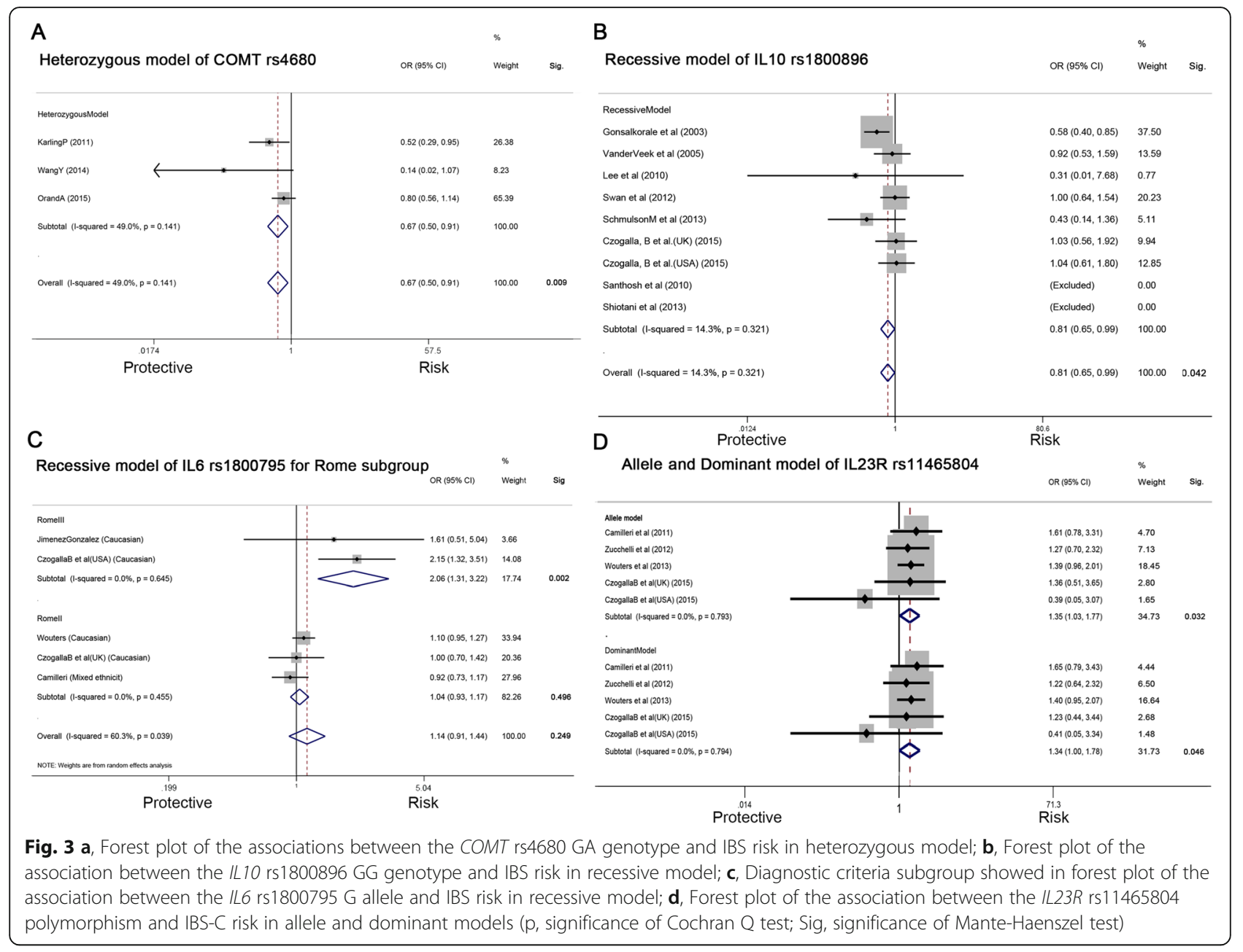

TNFSF15 rs4263839 and IBS risk

Four studies involving 2068 IBS patients and 1959 controls analyzed the association of TNFSF15 rs4263839 $(A>G)$ and IBS risk (Table 1). A significantly positive association betweenTNFSF15 rs4263839 polymorphism and IBS development was found in AM (G vs. A, OR = 5.139, 95\% CI: 3.859-6.844, $P<0.01)$, DM (GA + GG vs. $\mathrm{AA}, \mathrm{OR}=6.527,95 \% \mathrm{CI}: 4.616-9.229, P<0.01)$, HoM (GG vs. AA, OR $=19.127,95 \%$ CI: $15.395-23.765, P<$ 0.01 ) and $\mathrm{HeM}$ (GA vs. AA models, OR $=9.361,95 \% \mathrm{CI}$ : 4.702-18.637, $P<0.01)$. AM was used for subgroup analysis. As for IBS subtype (Fig. 4a and b), the G allele increased the risks for both IBS-C $(\mathrm{OR}=4.79,95 \% \mathrm{CI}$ : 4.16-5.51, $P<0.01)$ and IBS-D $(\mathrm{OR}=4.24,95 \% \mathrm{CI}$ : 3.74-4.81, $P<0.01)$. Moreover, subgroup analysis of Caucasian (Fig. 4a and b) also supported the results.

\section{TNFSF15 rs6478108 and IBS risk}

There were three studies involving 1527 IBS patients and 1008 controls that analyzed the association of
TNFSF15 rs6478108 (C > T) and IBS risk (Table 1). Polymorphism increases the risk of IBS in AM ( $\mathrm{T}$ vs. C, $\mathrm{OR}=1.043,95 \% \mathrm{CI}: 1.016-1.287, P=0.026)$ and HoM (TT vs. CC models, OR $=1.306,95 \%$ CI: $1.005-1.697$, $P=0.045)$ (Fig. 4c and d) accompany with good homogeneity (AM: $\mathrm{I}^{2}=20.3 \%, P=0.288$; HoM: $\mathrm{I}^{2}=20.5 \%, P=$ 0.287 ). Because all the subjects participating in these studies were Caucasian, only subgroup analysis of diagnostic criteria was performed, but the results suggested no correlations.

\section{SNPs had no association with IBS risk}

Eight studies involving 1868 IBS patients and 1462 controls were analyzed for the association of TNFQ rs1800629 (G > A), four studies involving 470 IBS patients and 485 controls were analyzed for the association of IL10 rs1800871 (T > C), four studies involving 724 IBS patients and 839 controls that analyzed the association of GNB3 rs5443 (C> T) and IBS risk (Table 1). Five genetic models were used for analysis, but no association of polymorphism with IBS risk was found in any 


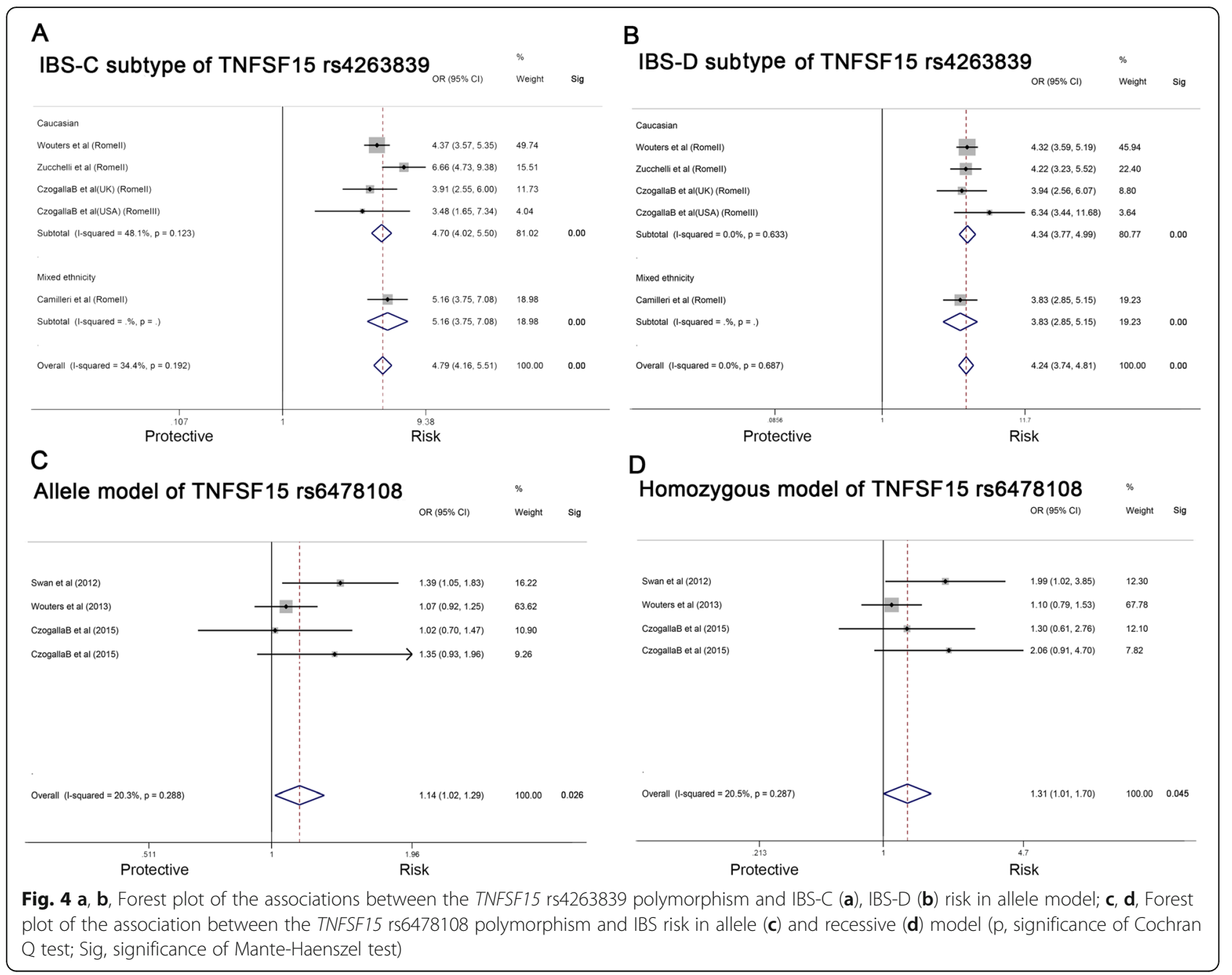

of the models. In addition, the AM was used for subgroup analysis, there was no association in the subgroup analysis.

\section{Discussion}

As a multi-pathogenesis disease, the genetic risk $[6,18]$ of IBS have been demonstrated in many studies. More than 65 candidate genes have been reported for IBS. Many new IBS associated SNPs was found through different strategies, for example, the genome-wide association studies (GWAS) [19-21]. However, consensus of the major IBS risk genes has been hard to reach. F. Bonfiglio et al. [22] carried out a GWAS meta-analysis of patients with IBS, they found SNPs in regulation of ion channel activity such as SCN5A and SI as the most plausible pathway affecting IBS. However, GWAS origin risk genes have not been successfully replicated in independent studies. Those IBS risk SNPs are mainly located in introns or UTR regions, which complicating the explanation of the gene functions to IBS pathygenesis. Moreover, most of the IBS GWAS analysis are population-based rather than identified as IBS cohort-based, which may cause variations. With the development of techniques, more newly detected SNPs were found related with the development of IBS in case-control studies. For example, SNPs of calcium-sensing receptor polymorphism (CaSR) [23] rs1801725 and adrenergic receptor (ADR) [24-26]. Nevertheless, there is no overview of all IBS-associated polymorphisms. Thus, this systemic review synthesized all the published SNPs studies of IBS through a strict metaanalysis, with the goal of objectively determining the relevance of genetic SNPs with IBS.

In this study, 10 relevant SNPs from 28 studies were evaluated. Many other SNPs which reported in less than three studies or had unclear allele frequency in articles were not included, even if they were latest reported. A study by Czogalla et al. [10] was included because Czogalla and their colleagues utilized two independent case-control cohorts (UK and USA cohorts) and identified risk SNPs separately. Thus, we considered these data as two cohorts in our analysis. Allele model and other four genotype model (DM, RM, HeM and HoM) were 
used to give an exhaustive analysis of the association. Except for ethnicity subgroup, diagnostic criteria and IBS subtype were also defined as another two subgroups which might assist to further analysis.

Cytokine gene polymorphisms are important because they might be associated with changes in cytokine profiles. It represent immune system dysregulation in IBS development. Among all the SNPs, TNFSF15 rs4263839 and TNFSF15 rs6478108 increased the risk of IBS. TNFSF15 encodes for TL1A, which is a tumor necrosis factor superfamily member expressed in different immune cells. It may trigger an immune response through Th17 cell [27] and play an important role in the development of many autoimmune and inflammatory diseases. Studies have demonstrated a close association between TL1A and IBD. Genetic analysis also confirmed that the TNFSF15 gene is a race-specific susceptibility gene for IBD $[28,29]$ and TL1A was up-regulated both in intestinal mucosal $\mathrm{T}$-cells and peripheral blood mononuclear cells of IBD patients [30]. Animal experiments showed that anti-TL1A antibody could reduce intestinal inflammation in chronic colitis [28]. According to the results of this study, TNFSF15 rs4263839 G allele increasing the risk of IBS. It was found in IBS patients and different IBS subtypes (IBS-C, IBS-D). TNFSF15 rs6478108 $\mathrm{T}$ allele increased IBS risk as well, but no association was found in subgroup analysis. This finding might provide a clue for the overlaps between IBD and IBS, and it might become a treatment target for IBS. For another Th17-associated pathway, IL23R interacts with IL23 to regulate the activity of immune cells and plays an important role in the inflammatory response against infection by bacteria and viruses. IL23R rs11465804, which associated with increasing risk of IBD [7], has also been reported in case-control studies and GWAS in patients with IBS. It was hypothesized [31] that IL23R gene variants increased the secretion of Th17 in patients, leading to a protective effect. In this study, IL23R rs11465804 G allele of IBS-C patients represented a protective effect. However, fewer studies focus on IL23R rs11465804, and its function on intestinal motility is unclear which needs further analysis.

IL6 has been reported increasing in the plasma of IBS patients. IL6 rs1800795 mutation $(\mathrm{C}>\mathrm{G})$ is associated with higher plasma concentrations of IL6 during immune activation [32]. Our finding is intriguing, IL6 rs1800795 G allele doubled the risk of Caucasian IBS patients which diagnosed by Rome III criteria but not Rome II criteria. It might because IBS diagnostic criteria changed greatly from Rome II to Rome III, the later defined different IBS subtypes based on Bristol scale, which purifying IBS patients from other functional gastroenterology diseases. For IL10 rs1800896, people with GG allele seem to have lower risks developing to IBS. This result is consistent with previous studies [13]. In addition, a few studies confirmed a decreased IL10 level in the serum and intestinal mucosa of IBS patients. Probiotics, such as Bifidobacterium and Lactococcus, can regulate IL10 level to reduce mucosal inflammation [32-34].

Serotonin is an important neurotransmitter both in the CNS and GI tract. It is reuptaken by SERT which encoded by the SLC6A4 gene to regulate serotonin concentration. Case-control studies on SLC6A4 5-HTTLPR were conducted to verify this hypothesis. Some studies demonstrated a positive association while others failed to confirm that [35]. Mohammed YA et al. reported $s$ allele of SLC6A4 5-HTTLPR reduced the risk of IBS in Asian population, while another meta-analysis [9] found $l$ allele uniquely associated with increased IBS-C risk. Data in this meta-analysis only represented that $l s$ genotype of SLC6A4 5-HTTLPR associated with increased risk of IBS in Mongoloid ethnicity. No association was found in other genetic models and further subgroup analysis. SLC6A4 5-HTTLPR with a short variation $(s)$ has been shown to decrease the activity of SERT which may accelerate intestinal peristalsis. However, studies took for analysis have significant heterogeneity, further analyses are necessary to confirm the results. Though, serotonin plays a key role in intestinal motility, sensitivity and endocrine systems, due to its wide distribution and nonspecific effect, serotonin can also be influenced by IBS subtype, ethnicity and many other factors. It is very difficult to support a strong relationship of serotonin-associated polymorphisms with IBS. Based on the studies above and our results, it is understandable that serotonin has been the earliest treatment target of IBS but with a little application. GA genotype of COMT rs 4680 was associated with decreased IBS risk. COMT is an enzyme involved in the degradation of catecholamine neurotransmitters. COMT rs4680 leads to the substitution of valine (Val) by methionine (Met), which decreases the enzyme activity and is associated with a lower pain sensitivity threshold [24]. However, our result is not consistent with previous studies $[36,37]$. One reason might be the limited studies with mixed ethnicity - only three studies were analyzed, but Mongoloid, Caucasian, and other ethnicities were all included. Another reason might be the different DNA sources. For example, Orand et al. [24] extracted DNA from saliva. No evidence for a contribution of GN 33 rs5443, TNF $\alpha$ rs1800629, and IL10 rs1800871 to IBS was found in this study, which is consistent with previous researches $[10,11,38]$.

Some limitations to this meta-analysis require careful consideration. First, due to the rigorous filtering criteria, limited data were available. Hence, other factors such as SNPs detecting methods, environmental factors and the source of healthy controls for comparison, which may 
also affect susceptibility to IBS, were not accounted for in the present study. Second, allele and genotype effect on IBS risk were both analyzed but no best genetic model was determined. Differ from those monogenous hereditary diseases, the pathogenesis of IBS is the result of the combination of both environmental and genetic factors. It's hard to tell whether someone will develop IBS by having a specific allele mutation. Moreover, multiple comparisons through different genetic models can increase the probability of false-positive outcome as well.

\section{Conclusions}

In this review, it was confirmed that TNFSF15 rs4263839 and TNFSF15 rs6478108 associated with increased IBS risk, while IL10 rs1800896 GG genotype associated with decreased IBS risk. Diagnostic criteria changes had influence on the association between IL6 rs1800795 and IBS risk. And IL23R rs11465804 might become a new target for IBS-C developing. According to these findings, it might offer some insights into gene functions affecting IBS susceptibility and some clues in IBS genetic analysis.

\section{Supplementary information}

Supplementary information accompanies this paper at https://doi.org/10. 1186/s12876-019-1084-z.

Additional file 1: Table S1. Summary of studied according to SNP: this table summarized all the SNPs which had been reported in IBS and shows its reference. Table S2. Study characteristics analysis: this table shows the most important information for the studies which take into analysis in this SMRA.

\section{Abbreviations}

$\mathrm{Cl}$ : Confidence interval; COMT: Catechol-O-methyltransferase; Gl: Gastrointestinal; GNB3: $\beta 3$ subunit of G-protein; GWAS: Genome-wide association studies; HWE: Hardy-Weinberg equilibrium; IBD: Inflammation bowel disease; IBS: Irritable bowel syndrome; IBS-C: Constipation predominant IBS; IBS-D: Diarrhea predominant IBS; IBS-M: Mixture of diarrhea and constipation IBS; IBS-U: Unsubtyped IBS; IL: Interleukin; MeSH: Medical subject headings; OR: Odds ratios; SERT: Serotonin transporter; SNPs: Single nucleotide polymorphisms; TNF: Tumor necrosis factor

\section{Acknowledgements}

We thank Dr. Yanyan Shi of the department of Epidemiology of Peking University Third Hospital for her help on statistical methods and literature retrieval.

\section{Authors' contributions}

Study concept, interpretation of data and critical revision: LD. Literatures review and data analysis: SZ, BW and QJ. Drafting of the manuscript: SZ. Obtained funding: LD. All authors read and approved the final manuscript.

\section{Funding}

The studies were supported by the "National Natural Science Foundation of China (81670491)" and "The Capital Health Research and Development of Special Found (2016-2-4093)" that does not have its role in the design of the study and collection, analysis, and interpretation of data and in writing the manuscript.
Availability of data and materials

The datasets used and/or analyzed during the current study are available from the corresponding author on reasonable request.

Ethics approval and consent to participate

Not applicable.

\section{Consent for publication}

Not applicable.

\section{Competing interests}

The authors declare that they have no competing interests.

Received: 1 April 2019 Accepted: 1 October 2019

Published online: 15 October 2019

References

1. Ford AC, Lacy BE, Talley NJ. Irritable bowel syndrome. New Engl J Med. 2017:376(26):2566-78.

2. Siah K, Gong X, Yang XJ, Whitehead WE, Chen M, Hou X, Pratap N, Ghoshal UC, Syam AF, Abdullah M, et al. Rome Foundation-Asian working team report: Asian functional gastrointestinal disorder symptom clusters. Gut. 2018;67(6):1071-7

3. Holtmann GJ, Ford AC, Talley NJ. Pathophysiology of irritable bowel syndrome. Lancet Gastroenterol Hepatol. 2016;1(2):133-46.

4. Lembo A, Zaman M, Jones M, Talley NJ. Influence of genetics on irritable bowel syndrome, gastro-oesophageal reflux and dyspepsia: a twin study. Aliment Pharmacol Ther. 2007:25(11):1343-50.

5. Kalantar JS, Locke GR, Zinsmeister AR, Beighley CM, Talley NJ. Familial aggregation of irritable bowel syndrome: a prospective study. Gut. 2003: 52(12):1703-7.

6. Waehrens R, Li X, Sundquist J, Sundquist K, Zoller B. Perinatal and familial risk factors for irritable bowel syndrome in a Swedish national cohort. Scand J Gastroenterol. 2018;53(5):559-66.

7. Brant SR, Okou DT, Simpson CL, Cutler DJ, Haritunians T, Bradfield JP, Chopra P, Prince J, Begum F, Kumar A, et al. Genome-wide association study identifies African-specific susceptibility loci in African Americans with inflammatory bowel disease. Gastroenterology. 2017;152(1):206-17.

8. Lin Z, Wang Z, Hegarty JP, Lin TR, Wang Y, Deiling S, Wu R, Thomas NJ, Floros J. Genetic association and epistatic interaction of the interleukin-10 signaling pathway in pediatric inflammatory bowel disease. World J Gastroenterol. 2017:23(27):4897-909.

9. Zhang ZF, Duan ZJ, Wang LX, Yang D, Zhao G, Zhang L. The serotonin transporter gene polymorphism (5-HTTLPR) and irritable bowel syndrome: a meta-analysis of 25 studies. BMC Gastroenterol. 2014;14:23.

10. Czogalla B, Schmitteckert S, Houghton LA, Sayuk GS, Camilleri M, Olivo-Diaz A, Spiller R, Wouters MM, Boeckxstaens G, Bermejo JL, et al. A meta-analysis of immunogenetic case-control association studies in irritable bowel syndrome. Neurogastroenterol Motil. 2015;27(5):717-27.

11. Pan ZG, Xiao C, Su DX. No association of G-protein beta polypeptide 3 polymorphism with irritable bowel syndrome: evidence from a metaanalysis. World J Gastroenterol. 2014;20(20):6345-52.

12. Beyder A, Mazzone A, Strege PR, Tester DJ, Saito YA, Bernard CE, Enders FT, Ek WE, Schmidt PT, Dlugosz A, et al. Loss-of-function of the voltage-gated Sodium Channel $\mathrm{Na}(\mathrm{V}) 1.5$ (Channelopathies) in patients with irritable bowel syndrome. Gastroenterology. 2014;146(7):1659-68.

13. Bashashati M, Rezaei N, Bashashati H, Shafieyoun A, Daryani NE, Sharkey KA, Storr M. Cytokine gene polymorphisms are associated with irritable bowel syndrome: a systematic review and meta-analysis. Neurogastroenterol Motil. 2012;24(12):1102-566.

14. Gonzalez JR, Carrasco JL, Dudbridge F, Armengol L, Estivill X, Moreno V. Maximizing association statistics over genetic models. Genet Epidemiol. 2008;32(3):246-54

15. Yamada R, Okada Y. An optimal dose-effect mode trend test for SNP genotype tables. Genet Epidemiol. 2009;33(2):114-27.

16. Ahn K, Haynes C, Kim W, Fleur RS, Gordon D, Finch SJ. The effects of SNP genotyping errors on the power of the Cochran-Armitage linear trend test for case/control association studies. Ann Hum Genet. 2007;71(Pt 2):249-61.

17. Xu T, Li X, Wang W, Hu P, Du F. Detection of publication bias in metaanalysis of dichotomous variable- egger test and Begg test. J Evid Based Med. 2009;03:181-4. 
18. Henstrom M, D'Amato M. Genetics of irritable bowel syndrome. Mol Cell Pediatr. 2016;3(1):7.

19. Ek WE, Reznichenko A, Ripke S, Niesler B, Zucchelli M, Rivera NV, Schmidt PT, Pedersen NL, Magnusson P, Talley NJ, et al. Exploring the genetics of irritable bowel syndrome: a GWA study in the general population and replication in multinational case-control cohorts. Gut. 2015;64(11):1774-82.

20. Holliday EG, Attia J, Hancock S, Koloski N, McEvoy M, Peel R, D'Amato M, Agreus L, Nyhlin H, Andreasson A, et al. Genome-wide association study identifies two novel genomic regions in irritable bowel syndrome. Am J Gastroenterol. 2014;109(5):770-2.

21. Bonfiglio F, Zheng T, Garcia-Etxebarria K, Hadizadeh F, Bujanda L, Bresso F, Agreus L, Andreasson A, Dlugosz A, Lindberg G, et al. Female-specific association between variants on chromosome 9 and self-reported diagnosis of irritable bowel syndrome. Gastroenterology. 2018;155(1):168-79.

22. Strege PR, Mazzone A, Bernard CE, Neshatian L, Gibbons SJ, Saito YA, Tester DJ, Calvert ML, Mayer EA, Chang L, et al. Irritable bowel syndrome patients have SCN5A channelopathies that lead to decreased NaV1.5 current and mechanosensitivity. Am J Physiol Gastrointest Liver Physiol. 2018;314(4): G494-503.

23. Romero P, Schmitteckert S, Wouters MM, Houghton LA, Czogalla B, Sayuk GS, Boeckxstaens GE, Guenther P, Holland-Cunz S, Niesler B. No association between the common calcium-sensing receptor polymorphism rs1801725 and irritable bowel syndrome. BMC Med Genet. 2015;16:110.

24. Orand A, Gupta A, Shih W, Presson AP, Hammer C, Niesler B, Heendeniya N, Mayer EA, Chang L. Catecholaminergic gene polymorphisms are associated with Gl symptoms and morphological brain changes in irritable bowel syndrome. PLoS One. 2015;10(8):e135910.

25. Choi YJ, Hwang SW, Kim N, Park JH, Oh JC, Lee DH. Association between SLC6A4 serotonin transporter gene Lainked polymorphic region and ADRA2A -1291C>G and irritable bowel syndrome in Korea. J Neurogastroenterol Motil. 2014;20(3):388-99.

26. Kim HJ, Camilleri M, Carlson PJ, Cremonini F, Ferber I, Stephens D, McKinzie S, Zinsmeister AR, Urrutia R. Association of distinct alpha (2) adrenoceptor and serotonin transporter polymorphisms with constipation and somatic symptoms in functional gastrointestinal disorders. Gut. 2004;53(6):829-37.

27. Dand N, Mucha S, Tsoi LC, Mahil SK, Stuart PE, Arnold A, Baurecht H, Burden $A D$, Callis DK, Chandran V, et al. Exome-wide association study reveals novel psoriasis susceptibility locus at TNFSF15 and rare protective alleles in genes contributing to type I IFN signalling. Hum Mol Genet. 2017;26(21):4301-13.

28. Li H, Song J, Niu G, Zhang H, Guo J, Shih DQ, Targan SR, Zhang X. TL1A blocking ameliorates intestinal fibrosis in the T cell transfer model of chronic colitis in mice. Pathol Res Pract. 2018;214(2):217-27.

29. Liu JZ, van Sommeren S, Huang H, Ng SC, Alberts R, Takahashi A, Ripke S, Lee JC, Jostins L, Shah T, et al. Association analyses identify 38 susceptibility loci for inflammatory bowel disease and highlight shared genetic risk across populations. Nat Genet. 2015;47(9):979-86.

30. Slebioda TJ, Bojarska-Junak A, Cyman M, Landowski P, Kaminska B, Celinski K, Kmiec Z. Expression of death receptor 3 on peripheral blood mononuclear cells differes in adult IBD patients and children with newly diagnosed IBD. Cytometry B Clin Cytom. 2017;92(2):165-9.

31. Schmechel S, Konrad A, Diegelmann J, Glas J, Wetzke M, Paschos E, Lohse P, Goke B, Brand S. Linking genetic susceptibility to Crohn's disease with Th17 cell function: IL-22 serum levels are increased in Crohn's disease and correlate with disease activity and IL23R genotype status. Inflamm Bowel Dis. 2008;14(2):204-12.

32. Frydecka D, Pawlowski T, Pawlak D, Malyszczak K. Functional polymorphism in the interleukin 6 (IL6) gene with respect to depression induced in the course of interferon-alpha and ribavirin treatment in chronic hepatitis patients. Arch Immunol Ther Exp. 2016;64(Suppl 1):169-75.

33. Compare D, Rocco A, Coccoli P, Angrisani D, Sgamato C, lovine B, Salvatore $U$, Nardone G. Lactobacillus casei DG and its postbiotic reduce the inflammatory mucosal response: an ex-vivo organ culture model of post-infectious irritable bowel syndrome. BMC Gastroenterol. 2017;17(1):53.

34. O'Mahony L, McCarthy J, Kelly P, Hurley G, Luo F, Chen K, O'Sullivan GC, Kiely B, Collins JK, Shanahan F, et al. Lactobacillus and bifidobacterium in irritable bowel syndrome: symptom responses and relationship to cytokine profiles. Gastroenterology. 2005;128(3):541-51.

35. Katsumata R, Shiotani A, Matsumoto H, Fujita M, Haruma K. Tu1790 TPH1 and 5-HTT Gene Polymorphisms Affect Quality of Life of the Patients With
Diarrhea-Predominant Irritable Bowel Syndrome. Gastroenterology. 2016; 150(4, Supplement 1):S947-8.

36. Karling P, Danielsson A, Wikgren M, Soderstrom I, Del-Favero J, Adolfsson R, Norrback KF. The relationship between the val158met catechol-Omethyltransferase (COMT) polymorphism and irritable bowel syndrome. PLoS One. 2011;6(3):e18035.

37. Wang Y, Wu Z, Qiao H, Zhang Y. A genetic association study of single nucleotide polymorphisms in GNbeta3 and COMT in elderly patients with irritable bowel syndrome. Med Sci Monit. 2014;20:1246-54.

38. Qin SY, Jiang HX, Lu DH, Zhou Y. Association of interleukin-10 polymorphisms with risk of irritable bowel syndrome: a meta-analysis. World J Gastroenterol. 2013;19(48):9472-80.

\section{Publisher's Note}

Springer Nature remains neutral with regard to jurisdictional claims in published maps and institutional affiliations.

\section{Ready to submit your research? Choose BMC and benefit from:}

- fast, convenient online submission

- thorough peer review by experienced researchers in your field

- rapid publication on acceptance

- support for research data, including large and complex data types

- gold Open Access which fosters wider collaboration and increased citations

- maximum visibility for your research: over $100 \mathrm{M}$ website views per year

At BMC, research is always in progress.

Learn more biomedcentral.com/submissions 